\title{
Perfil epidemiológico de malformações arteriovenosas encefálicas em uma série monocêntrica
}

\author{
Danilo Marques Nogueira1, Aron Athaide Diniz', Renato de Amorin Motta Deusdará1, \\ Iruena Moraes Kessler ${ }^{2}$, Nelson Geraldo Freire Neto ${ }^{3}$ \\ Hospital de Base do Distrito Federal, Brasília, DF, Brasil e Hospital Universitário de Brasília, Brasília, DF, Brasil.
}

\section{RESUMO}

Objetivo: Avaliar o perfil epidemiológico dos pacientes atendidos na Unidade de Neurocirurgia do Hospital de Base do Distrito Federal (HBDF), unidade de referência de doenças cerebrovasculares no Distrito Federal, bem como os métodos multidisciplinares de tratamento utilizados e a evolução clínica. Métodos: Realizou-se a análise retrospectiva de 42 pacientes portadores de malformações arteriovenosas (MAV) encefálicas admitidos no Serviço de Neurocirurgia do HBDF entre agosto de 2007 e janeiro de 2012, tratados conservadoramente ou submetidos a algum tipo de intervenção: microcirurgia intracraniana, embolização, técnica combinada ou radiocirurgia. Resultados: A distribuição por gênero perfez uma relação de 1,1:1,0 (masculino:feminino). Houve predomínio da localização supratentorial (85\%, 36/42). Conforme a Escala de Spetzler-Martin (ESM), evidenciou-se predomínio de graus intermediários (graus II, III e IV), totalizando 96\% (40/42). Dez pacientes (24\%, 10/42) foram submetidos a tratamento cirúrgico convencional, 22 pacientes $(52 \%, 22 / 42)$ à embolização (tratamento endovascular), 1 paciente $(2,38 \%, 1 / 42)$ a tratamento combinado (endovascular e cirurgia), 1 (2,38\%, $1 / 42)$ à radiocirurgia e 8 pacientes $(19,05 \%, 8 / 42)$ foram tratados conservadoramente. A evolução clínica caracterizada pela Escala Modificada de Rankin (EMR) mostrou semelhança estatística em relação aos diversos tratamentos estabelecidos. Conclusão: As MAVs são lesões congênitas que, apesar de baixa prevalência, são alvo importante de estudo, por acometer principalmente adultos jovens e em idade produtiva. Este estudo, realizado em um centro de referência do Distrito Federal, evidencia características demográficas peculiares dessas lesões, que são compatíveis com dados prévios da literatura e mostra que a abordagem terapêutica multidisciplinar é essencial para seu correto tratamento.

\section{PALAVRAS-CHAVE}

Malformações arteriovenosas, hemorragia cerebral, neurocirurgia, embolização terapêutica, radiocirurgia.

\section{ABSTRACT}

Epidemiologic profile of brain arteriovenous malformation in a monocentric series

Objective: To assess the epidemiological profile of patients treated in the Neurosurgery Unit of the Hospital de Base of the Federal District (HBDF) reference unit of cerebrovascular diseases in the Federal District, as well as multidisciplinary treatment methods used and the clinical outcome. Methods: We performed a retrospective analysis of 42 patients with arteriovenous malformations (AVMs) in the brain admitted at Neurosurgery Service of HBDF between August 2007 and January 2012, treated conservatively or undergoing some type of intervention: intracranial microsurgery, embolization, combined technique or radiosurgery. Results: The gender distribution amounted a ratio of 1.1:1.0 (male:female). Predominated supratentorial localization (85\%, 36/42). According to the Spetzler-Martin Scale (ESM), we observed a predominance of intermediate grades (grades II, III and IV) totaling 96\% (40/42). Ten patients $(24 \%, 10 / 42)$ were subjected to conventional surgical treatment, 22 patients $(52 \%, 22 / 42)$ to embolization (endovascular treatment), 1 patient $(2.38 \%, 1 / 42)$ the combined treatment (endovascular and surgery), $1(2.38 \%, 1 / 42)$ radiosurgery and 8 patients $(19.05 \% 8 / 42)$ were treated conservatively. The clinical evolution characterized by the modified Rankin Scale (EMR) showed statistical similarity with respect to several established treatments. Conclusion: The AVMs are congenital lesions that despite low prevalence are important target of study, since it affects mostly young adults and working age. This study, conducted in a referral center in the Federal District, shows peculiar demographic characteristics of these lesions, which are consistent with previous literature data and shows that the multidisciplinary therapeutic approach is essential for its proper treatment.

\section{KEYWORDS}

Arteriovenous malformations, cerebral hemorrhage, neurosurgery, embolization therapeutic, radiosurgery.

1 Médico-residente em Neurocirurgia do Hospital de Base do Distrito Federal, Brasília, DF, Brasil.

2 Professora adjunta, chefe da Unidade de Neurocirurgia do Hospital Universitário de Brasília, Brasília, DF, Brasil.

3 Neurocirurgião e supervisor do Programa de Residência Médica de Neurocirurgia do Hospital de Base do Distrito Federal, Brasília, DF, Brasil. 


\section{Introdução}

Malformações arteriovenosas (MAV) são lesões congênitas decorrentes do desenvolvimento anômalo vascular da circulação fetal, ocorrendo a persistência de vasos primitivos com ausência de capilares perfazendo uma comunicação direta entre artérias e veias. ${ }^{1}$

Desde sua primeira descrição, há mais de um século, as malformações arteriovenosas (MAV) do encéfalo são conhecidas causas de morbimortalidade. ${ }^{2}$ Essas são lesões vasculares raras com incidência de aproximadamente um caso por 100.000 pessoas-ano. ${ }^{3,4}$ Porém, a incidência de lesões não rotas e assintomáticas vem crescendo gradualmente em decorrência da facilidade e disponibilidade de exames de imagem de melhor qualidade. $^{3}$

Quando sintomáticas, as formas de apresentação mais comuns ainda são os acidentes vasculares encefálicos hemorrágicos (AVEH), seguidos por crises epilépticas, cefaleia e déficits neurológicos focais. ${ }^{3,5}$

Como opções de tratamento existem a microcirurgia, a embolização, a radiocirurgia estereotáxica e a combinação dos métodos. ${ }^{6}$ Há, ainda, a opção de tratamento conservador, porém é importante lembrar que o risco de ruptura da MAV não tratada é de $2 \%$ a $4 \%$ ao ano de acordo com suas características citadas anteriormente. ${ }^{3,6-8}$

O tratamento deve ser definido a partir de características da MAV, como tamanho, localização e configuração da drenagem venosa, que se classificam na escala de Spetzler e Martin, ${ }^{9}$ além de associação com aneurismas de fluxo e alta pressão sanguínea intranidal. ${ }^{5,6}$

O Hospital de Base do Distrito Federal (HBDF) é o centro de referência para doenças neurocirúrgicas no Distrito Federal. No entanto, não há relatos sobre as características demográficas de MAVs encefálicas atendidas neste serviço. Este estudo tem como objetivo traçar um perfil epidemiológico da população de pacientes portadores de MAV encefálicas atendidas no HBDF entre agosto de 2007 e janeiro de 2012, visando identificar variáveis de risco para sangramento e peculiaridades que possam aperfeiçoar o manejo dessa enfermidade.

\section{Material e métodos}

\section{Amostra demográfica e apresentação clínica}

Entre agosto de 2007 e janeiro de 2012, foram admitidos 5.724 pacientes no Serviço de Neurocirurgia do Hospital de Base do Distrito Federal (HBDF). Quarenta e dois (42) pacientes eram portadores de malformações arteriovenosas (MAV) encefálicas (42/5.754, prevalência $0,76 \%$ ). Esses 42 pacientes foram analisados retrospectivamente após aprovação do comitê de ética do HBDF, utilizando-se dados do prontuário médico. Foram 22 homens e 20 mulheres com idade média entre 28 anos e 35 anos (média de 31 anos). Em 29 (69\%, 29/42) os sintomas foram causados por hemorragia intracraniana e as manifestações clínicas mais prevalentes foram cefaleia $(86 \%, 36 / 42)$, sendo aguda em 30 $(71 \%, 30 / 42)$ e crônica em seis $(14 \%, 6 / 42)$, vômitos em $10(24 \%, 10 / 42)$, confusão mental em três $(7 \%, 3 / 42)$, crises convulsivas em $13(31 \%, 13 / 42)$, ataxia em dois $(5 \%, 2 / 42)$, déficit focal em um $(2 \%, 1 / 42)$ e diminuição do nível de consciência em 13 casos $(31 \%, 13 / 42)$.

\section{Classificação e localização das MAVS}

As $42 \mathrm{MAVs}$ foram classificadas conforme a escala de Spetzler e Martin ${ }^{9}$ (SM), sendo um caso grau I ( $2 \%$, $1 / 42), 12$ grau II $(28 \%, 12 / 42), 18$ grau III $(42 \%, 18 / 42)$, 10 grau IV $(23 \%, 10 / 42)$ e um caso grau V $(2 \%, 1 / 42)$. As MAVs estavam em localização supratentorial em 36 casos $(85 \%, 36 / 42)$, infratentorial em cinco $(11 \%, 5 / 42)$ e nos dois sítios em um $(2 \%, 1 / 42)$.

\section{Estratégias de tratamento}

O Serviço de Neurocirurgia do HBDF tem como primeira opção no tratamento de MAVs a cirurgia convencional para as MAVs Spetzler-Martin I e II. Os casos III, IV e V são considerados para tratamento endovascular ou conservador. O tempo de tratamento cirúrgico era determinado conforme a apresentação clínica. Casos que necessitavam de craniotomia descompressiva de urgência pela presença de hematoma intraparenquimatoso ou intraventricular eram tratados agudamente. A embolização ou o tratamento endovascular (TEV) eram definidos após 21 dias a três meses (média 56 dias) após o diagnóstico e reabsorção do hematoma. Todos os pacientes realizaram tomografia computadorizada de crânio à admissão e angiografia cerebral digital.

\section{Seguimento clínico - Follow up}

O seguimento clínico foi precoce e documentado no momento da alta hospitalar utilizando-se a Escala Modificada de Rankin ${ }^{10}$ (EMR).

\section{Análise estatística}

Para fins de mapeamento epidemiológico foram utilizadas variáveis como sexo, presença ou ausência de 
sangramento, localização da malformação vascular encefálica, manifestações clínicas à admissão e tratamento proposto (cirúrgico, embolização, técnica combinada, radiocirurgia ou tratamento conservador).

Os dados obtidos foram analisados utilizando-se do programa "Software R" (http://www.r-project. org $/)^{11}$ para avaliar a relação entre as diversas variáveis de interesse relacionadas e a associação destas com as escalas e graduações referidas anteriormente (Spetzler-Martin e Rankin).

Os testes utilizados pelo programa "Software R" se restringiram no teste Qui-Quadrado, na comparação de médias, no teste de Mann-Whitney e no teste de Kruskal-Wallis. ${ }^{11}$

\section{Resultados}

\section{Risco de sangramento}

\section{GÊNERO}

Dos 42 pacientes, 22 eram do sexo masculino e 20, do sexo feminino, perfazendo uma relação de 1,1:1,0. Não houve correlação entre gênero masculino ou feminino e o risco de sangramento $(\mathrm{p}=0,58 ; \mathrm{p}>0,05)$ (Tabela 1).

\begin{tabular}{|c|c|c|c|}
\hline \multirow{2}{*}{ Gênero } & \multicolumn{2}{|c|}{ Sangramento } & \multirow{2}{*}{ Total } \\
\hline & Sim & Não & \\
\hline Masculino & $16(38,1 \%)$ & $6(14,29 \%)$ & 22 \\
\hline Feminino & $13(30,95 \%)$ & $7(16,67 \%)$ & 20 \\
\hline Total & 29 & 13 & 42 \\
\hline
\end{tabular}

\section{Localização}

Houve predomínio de localização supratentorial evidenciada em 31 casos $(85 \%, 36 / 42)$, sendo infratentorial em cinco casos $(11 \%, 5 / 42)$ e um $(2 \%, 1 / 42)$ nos dois sítios. Quando comparado o risco de sangramento, a localização supratentorial mostrou-se com risco significativamente maior $(\mathrm{p}<0,05)$ (Tabela 2$)$.

\begin{tabular}{lccc}
\hline \multicolumn{3}{c}{$\begin{array}{c}\text { Tabela 2 - Correlação entre presença de } \\
\text { sangramento e localização }\end{array}$} \\
\cline { 2 - 3 } Localização & \multicolumn{2}{c}{ Sangramento } & Total \\
\cline { 2 - 4 } & Sim & Não & 36 \\
\hline Supratentorial & $27(64,2 \%)$ & $9(21,4 \%)$ & 5 \\
Infratentorial & $2(4,7 \%)$ & $3(7,1 \%)$ & 1 \\
Supra e infra & 0 & $1(2,3 \%)$ & 42 \\
Total & 29 & 13 & 5 \\
\hline
\end{tabular}

\section{Escala de Spetzler-Martin (ESM)}

Conforme a Classificação de Spetzler-Martin, foram evidenciados um caso grau I $(2 \%, 1 / 42), 12$ casos grau II $(28 \%, 12 / 42), 18$ casos grau III $(42 \%, 18 / 42), 10$ casos grau IV $(23 \%, 10 / 42)$ e um caso grau V $(2 \%, 1 / 42)$ (Tabela 3 e Figura 1).

\begin{tabular}{|c|c|c|c|}
\hline \multicolumn{4}{|c|}{$\begin{array}{c}\text { Tabela } 3 \text { - Correlação entre sangramento } \\
\text { e Spetzler-Martin à admissão }\end{array}$} \\
\hline \multirow{2}{*}{ Variáveis } & \multicolumn{2}{|c|}{ Sangramento } & \multirow{2}{*}{ Total } \\
\hline & Sim & Não & \\
\hline \multicolumn{4}{|l|}{ Spetzler } \\
\hline I & $1(2,38 \%)$ & 0 & 1 \\
\hline II & $10(23,81 \%)$ & $2(4,76 \%)$ & 12 \\
\hline III & $10(23,81 \%)$ & $8(19,05 \%)$ & 18 \\
\hline IV & $7(16,67 \%)$ & $3(7,14 \%)$ & 10 \\
\hline V & $1(2,38 \%)$ & 0 & 1 \\
\hline
\end{tabular}

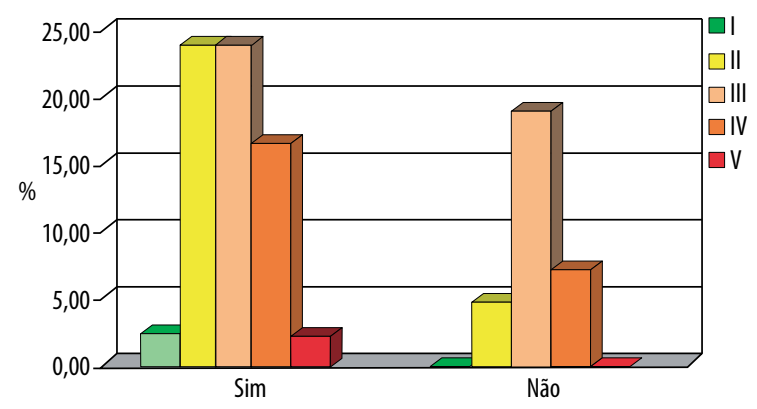

Figura 1 - Distribuição de Spetzler-Martin em relação à presença de sangramento.

\section{Tratamento}

Dez pacientes $(24 \%, 10 / 42)$ foram submetidos a tratamento cirúrgico convencional, 22 pacientes $(52 \%$, 22/42) a tratamento endovascular (embolização), um paciente $(2,38 \%, 1 / 42)$ a tratamento combinado (endovascular e cirurgia), um $(2,38 \%, 1 / 42)$ a radioterapia e oito $(19,05 \%, 8 / 42)$ foram tratados conservadoramente. Houve predomínio significativo de casos com ESM II para tratamento cirúrgico, oito casos $(80 \%, 8 / 10)$ e ESM III e IV para TEV com 19 casos $(86 \%, 19 / 22)$ $(\mathrm{p}<0,05)$ (Tabela 4).

\section{Evolução clínica}

A mortalidade total foi de $2 \%$ (1/42), Rankin 6, em um paciente tratado conservadoramente.

Dos 10 pacientes tratados com cirurgia, cinco $(50 \%$, $5 / 10)$ evoluíram com EMR de 0, quatro $(40 \%, 4 / 10)$ com EMR de 1, e um $(10 \%, 1 / 10)$ com EMR de $2(10 \%$, $1 / 10)$. Entre os 22 pacientes tratados com TEV, treze 
$(59 \%, 13 / 22)$ evoluíram com EMR de 0 , sete $(32 \%, 7 / 22)$ com EMR de 1, um $(4,5 \%, 1 / 22)$ com EMR de 2 e um $(4,5 \%, 1 / 22)$ com EMR de 5.

Dos oito $(19 \%, 8 / 42)$ pacientes do grupo tratado conservadoramente, cinco $(62,5 \%, 5 / 8)$ evoluíram com EMR de 0, dois $(25 \%, 2 / 8)$ com EMR de 1 e um $(12,5 \%$, 1/8) com EMR de 6. Um caso tratado com técnica combinada (cirurgia e TEV) evoluiu com EMR de 4. Não houve diferença estatística entre os grupos ( $\mathrm{p}>$ 0,05) (Tabela 5).

O paciente submetido à radiocirurgia não foi acompanhado em nosso serviço, porém sua graduação na EMR era de 2 quando foi encaminhado para realização deste procedimento em outro centro.

\section{Complicações}

Entre as complicações relatadas na admissão e durante a internação hospitalar, evidenciaram-se presença de sangramento intraventricular em 16\% (7/42), sangramento intraparenquimatoso em $2 \%(1 / 42)$, hematoma subdural agudo após TEV em 2\% (1/42), sangramento da MAV durante o TEV em 2\% (1/42), infecção urinária em $2 \%(1 / 42)$ em um paciente submetido à cirurgia e hidrocefalia em $4 \%$ (2/42), sendo que um desses evoluiu com infecção do sistema ventricular cerebral (ventriculite).

\section{Discussão}

Malformações arteriovenosas (MAV) são lesões congênitas decorrentes do desenvolvimento anômalo vascular da circulação fetal, ocorrendo a persistência de vasos primitivos com ausência de capilares perfazendo uma comunicação direta entre artérias e veias. ${ }^{1}$

As MAV são lesões infrequentes. ${ }^{3} \mathrm{O}$ presente estudo está em consonância com esse dado da literatura, considerando prevalência de $0,76 \%$ nos quatro anos estudados em relação à população geral atendida no Serviço de Neurocirurgia do HBDF, centro de referência no DF. No entanto, esse número tende a crescer com a melhoria dos métodos de diagnóstico e acesso ao serviço médico terciário.

Quanto aos aspectos demográficos, as MAVs podem apresentar peculiaridades, como descritas por van Beijnum et al. ${ }^{6}$, em que o sexo masculino estaria associado a baixo risco de sangramento e morbimortalidade. No entanto, o presente estudo não mostrou correlação significativa entre sexo e risco de hemorragia $(\mathrm{p}=0,58$; $\mathrm{p}>0,05)$ (Tabela 1).

De acordo com a classificação de Spetzler-Martin, mostrou-se predominância de graduações intermediárias (grau II, III e IV), e esses graus intermediários tiveram maior risco de ruptura conforme visualizado na figura 1. As associações sexo e sangramento, Spet-

\begin{tabular}{|c|c|c|c|c|c|c|}
\hline \multirow{2}{*}{ Variáveis } & \multicolumn{5}{|c|}{ Spetzler } & \multirow{2}{*}{ Total } \\
\hline & I & II & III & IV & $\mathrm{V}$ & \\
\hline \multicolumn{7}{|l|}{ Procedimento } \\
\hline Cirurgia & $1(2,38 \%)$ & $8(19,05 \%)$ & $1(2,38 \%)$ & 0 & 0 & 10 \\
\hline Conservador & 0 & $1(2,38 \%)$ & $5(11,9 \%)$ & $2(4,76 \%)$ & 0 & 8 \\
\hline Embolização & 0 & $2(4,76 \%)$ & $12(28,57 \%)$ & $7(16,66 \%)$ & $1(2,38 \%)$ & 22 \\
\hline $\begin{array}{l}\text { Embolização } \\
+ \text { Cirurgia }\end{array}$ & 0 & $1(2,38 \%)$ & 0 & & 0 & 1 \\
\hline Radiocirurgia & 0 & 0 & 0 & $1(2,38 \%)$ & 0 & 1 \\
\hline
\end{tabular}

\begin{tabular}{|c|c|c|c|c|c|c|c|}
\hline \multirow{2}{*}{ Variáveis } & \multicolumn{6}{|c|}{ Rankin } & \multirow{2}{*}{ Total } \\
\hline & 1 & 2 & 3 & 4 & 5 & 6 & \\
\hline \multicolumn{8}{|l|}{ Procedimento } \\
\hline Cirurgia & $5(11,9 \%)$ & $4(9,5 \%)$ & $1(2,4 \%)$ & 0 & 0 & 0 & 10 \\
\hline Conservador & $5(11,9 \%)$ & $2(4,7 \%)$ & 0 & 0 & 0 & $1(2,4 \%)$ & 8 \\
\hline Embolização & $13(30,9 \%)$ & $7(16,6 \%)$ & $1(2,4 \%)$ & 0 & $1(2,4 \%)$ & 0 & 22 \\
\hline $\begin{array}{l}\text { Embolização } \\
\text { + Cirurgia }\end{array}$ & 0 & 0 & 0 & $1(2,4 \%)$ & 0 & 0 & 1 \\
\hline Radiocirurgia & 0 & $1(2,4 \%)$ & 0 & 0 & 0 & 0 & 1 \\
\hline
\end{tabular}


zler e sangramento, Rankin e sangramento, Rankin e procedimento se mostraram independentes $(p>0,05)$.

Considerando a apresentação clínica, podem ser lesões assintomáticas e normalmente descobertas ao acaso durante a realização de exames de neuroimagem. ${ }^{2}$ Quando sintomáticas, são habitualmente secundárias à hemorragia, predominando cefaleia, crises convulsivas, redução do nível de consciência e déficit motor. Laakso e Hernesniemi ${ }^{3}$ relataram cerca de 45 a $72 \%$ dos pacientes com hemorragia e 18 a $35 \%$ com epilepsia. Podem se manifestar em diversas idades, com predomínio de adultos jovens..$^{12} \mathrm{~A}$ forma hemorrágica é a mais frequente no meio hospitalar e a descoberta fortuita é prevalente em ambiente ambulatorial. . $3,3,-7,12^{-12}$

O presente estudo corrobora esses dados, levando-se em conta que os casos foram documentados em ambiente hospitalar, com 29 casos $(69 \%, 29 / 42)$ de sintomas secundários à hemorragia intracraniana, sendo as manifestações clínicas mais prevalentes: cefaleia (86\%, $36 / 42)$, sendo aguda em 30 (71\%, 30/42) e crônica em seis (14\%, 6/42); vômitos em 10 (24\%, 10/42), confusão mental em três $(7 \%, 3 / 42)$, crises convulsivas em 13 $(31 \%, 13 / 42)$, ataxia em dois $(5 \%, 2 / 42)$, déficit focal em um $(2 \%, 1 / 42)$ e diminuição do nível de consciência em 13 casos $(31 \%, 13 / 42)$.

O tratamento das MAVs ainda é alvo de grandes discussões, levando-se em conta o risco-benefício que as técnicas atuais oferecem (cirurgia, embolização, radiocirurgia). Este deve ser indicado tendo em vista peculiaridades da lesão que apresentem maior risco de hemorragia, justificando o risco de uma intervenção. ${ }^{3,6}$

Habitualmente, são designadas para o tratamento cirúrgico as MAVs com SM de I e II, considerando particularidades anatômicas favoráveis a esse tipo de intervenção. ${ }^{6}$ Graus mais elevados na ESM, III a V, são por consenso atual encaminhados à embolização. ${ }^{6}$ A radiocirurgia é, em casos selecionados, comumente indicada a lesões menores que $3,5 \mathrm{~cm}$ e/ou de difícil acesso, como lesões pontinas e de núcleos da base. ${ }^{6} \mathrm{Na}$ presente série, conforme documentado na tabela 3 , $69 \%$ dos pacientes apresentaram MAVs com SM de graus elevados (III, IV, V) e, portanto, houve predomínio significativo de tratamento endovascular com $52 \%$ da amostra submetida à embolização $(\mathrm{p}<0,05)$. Esse dado refere uma evolução referente ao manuseio dessas lesões por equipes multidisciplinares, formadas por neurologistas, neurocirurgiões e neuroradiologistas intervencionistas.

A mortalidade total foi de $2 \%$ (1/42), em paciente do grupo tratado conservadoramente, o que está de acordo com dados já reportados. Ao se considerar a evolução clínica utilizando a EMR e o tipo de tratamento utilizado conforme relatado na tabela 5 , não houve diferença significativa entre os grupos, apesar da heterogeneidade destes $(\mathrm{P}>0,05)$.
Este estudo tem diversas limitações, como amostra reduzida, falha no seguimento tardio, documentação de prontuários heterogêneos e dados radiológicos ausentes ou não padronizados. No entanto, trata-se de uma tentativa inicial de documentar características demográficas dessa população, com a finalidade de identificar fatores que possam aperfeiçoar o atendimento.

\section{Conclusão}

As MAVs são lesões congênitas que, apesar de baixa prevalência, são alvo importante de estudo, por acometer principalmente adultos jovens e em idade produtiva. O presente estudo epidemiológico transversal, realizado em um centro de referência do Distrito Federal, mostrou características demográficas peculiares dessas lesões, que são compatíveis com dados prévios da literatura, e evidencia que a abordagem terapêutica multidisciplinar é essencial para seu correto tratamento.

Considerando as limitações inerentes ao método empregado, a amostra reduzida e o seguimento prejudicado, são necessários estudos prospectivos mais elaborados sobre o tema.

\section{Agradecimentos}

Os autores agradecem a todos servidores do HBDF que, direta ou indiretamente, tornaram possível a realização deste trabalho. De modo especial, à equipe da Secretaria da Unidade de Neurocirurgia e à equipe do Arquivo Físico do HBDF.

\section{Conflitos de interesse}

Os autores declaram não haver conflitos de interesse.

\section{Referências}

1. Mullan S, Mojtahedi S, Johnson DL, Macdonald RL. Embryological basis of some aspects of cerebral vascular fistulas and malformations. J Neurosurg. 1996;85(1):1-8.

2. Al-Shahi R, Warlow C. A systematic review of the frequency and prognosis of arteriovenous malformations of the brain in adults. Brain. 2001;124:1900-26. 
3. Laakso A, Hernesniemi J. Arteriovenous malformations: epidemiology and clinical presentation. Neurosurg Clin N Am. 2012;23(1):1-6.

4. ApSimon HT, Reef H, Phadke RV, Popovic EA. A populationbased study of brain arteriovenous malformation: long-term treatment outcomes. Stroke. 2002;33(12):2794-800.

5. Stapf C, Mohr JP, Pile-Spellman J, Solomon RA, Sacco RL, Connolly ES Jr. Epidemiology and natural history of arteriovenous malformations. Neurosurg Focus. 2001;11(5):e1.

6. van Beijnum J, van der Worp HB, Buis DR, Al-Shahi Salman R, Kappelle LJ, Rinkel GJ, et al. Treatment of brain arteriovenous malformations: a systematic review and meta-analysis. JAMA. 2011;306(18):2011-9.

7. Brown RD Jr, Wiebers DO, Torner JC, O'Fallon WM. Frequency of intracranial hemorrhage as a presenting symptom and subtype analysis: a population-based study of intracranial vascular malformations in Olmsted Country, Minnesota. J Neurosurg. 1996;85(1):29-32.

8. Mast H, Young WL, Koennecke HC, Sciacca RR, Osipov A, Pile-Spellman J, et al. Risk of spontaneous haemorrhage after diagnosis of cerebral arteriovenous malformation. Lancet. 1997;350(9084):1065-8.

9. Spetzler RF, Martin NA. A proposed grading system for arteriovenous malformations. J Neurosurg. 1986;65(4):47683.

10. Bonita R, Beaglehole R. Recovery of motor function after stroke. Stroke. 1988;19(12):1497-500.

11. The $\mathrm{R}$ Project for statistical computing. Disponível em: http://www.r-project.org/. Acesso em: $1^{\circ}$ jul, 2013.

12. Hofmeister C, Stapf C, Hartmann A, Sciacca RR, Mansmann $\mathrm{U}$, terBrugge $\mathrm{K}$, et al. Demographic, morphological, and clinical characteristics of 1289 patients with brain arteriovenous malformation. Stroke. 2000;31(6):1307-10.

Endereço para correspondência

Danilo Marques Nogueira

Av. B, esquina com Rua 9A, 164, ap. 1303

Ed. Constelation, Setor Oeste

74110-030 - Goiânia, GO, Brasil

E-mail: danilomnogueira@yahoo.com.br 